\title{
Complete genome sequence of the moderate thermophile Anaerobaculum mobile type strain (NGA $\left.{ }^{\top}\right)$
}

\author{
Konstantinos Mavromatis ${ }^{1}$, Erko Stackebrandt ${ }^{2}$, Brittany Held ${ }^{1,3}$, Alla Lapidus ${ }^{1}$, Matt Nolan ${ }^{1}$, \\ Susan Lucas ${ }^{1}$, Nancy Hammon ${ }^{1,3}$, Shweta Deshpande ${ }^{1,3}$, Jan-Fang Cheng ${ }^{1}$, Roxanne Tapia ${ }^{1,3}$, \\ Lynne A. Goodwin ${ }^{1,3}$, Sam Pitluck ${ }^{1}$, Konstantinos Liolios ${ }^{1}$, Ioanna Pagani ${ }^{1}$, Natalia Ivanova ${ }^{1}$, \\ Natalia Mikhailova', Marcel Huntemann', Amrita Pati', Amy Chen ${ }^{4}$, Krishna Palaniappan ${ }^{4}$, \\ Miriam Land ${ }^{1,5}$, Manfred Rohde ${ }^{6}$, Stefan Spring ${ }^{2}$, Markus Göker ${ }^{2}$, Tanja Woyke ${ }^{1}$, John C. \\ Detter $^{3}$, James Bristow ${ }^{1}$, Jonathan A. Eisen ${ }^{1,7}$, Victor Markowitz ${ }^{4}$, Philip Hugenholtz ${ }^{1,8}$, Hans- \\ Peter Klenk $^{2 *}$, and Nikos C. Kyrpides ${ }^{1}$ \\ ${ }^{1}$ DOE Joint Genome Institute, Walnut Creek, California, USA \\ ${ }^{2}$ Leibniz-Institute DSMZ - German Collection of Microorganisms and Cell Cultures, \\ Braunschweig, Germany \\ ${ }^{3}$ Los Alamos National Laboratory, Bioscience Division, Los Alamos, New Mexico, USA \\ ${ }^{4}$ Biological Data Management and Technology Center, Lawrence Berkeley National \\ Laboratory, Berkeley, California, USA \\ ${ }^{5}$ Oak Ridge National Laboratory, Oak Ridge, Tennessee, USA \\ ${ }^{6} \mathrm{HZI}$ - Helmholtz Centre for Infection Research, Braunschweig, Germany \\ ${ }^{7}$ University of California Davis Genome Center, Davis, California, USA \\ ${ }^{8}$ Australian Centre for Ecogenomics, School of Chemistry and Molecular Biosciences, The \\ University of Queensland, Brisbane, Australia
}

${ }^{*}$ Corresponding author: Hans-Peter Klenk

Keywords: Gram-negative, rod-shaped, motile, flagellum, non-spore forming, anaerobic, chemoorganotrophic, crotonate-reducer, Synergistetes, Synergistaceae, GEBA

Anaerobaculum mobile Menes and Muxí 2002 is one of three described species of the genus Anaerobaculum, family Synergistaceae, phylum Synergistetes. This anaerobic and motile bacterium ferments a range of carbohydrates and mono- and dicarboxylic acids with acetate, hydrogen and $\mathrm{CO}_{2}$ as end products. A. mobile $\mathrm{NGA}^{\top}$ is the first member of the genus Anaerobaculum and the sixth member of the phylum Synergistetes with a completely sequenced genome. Here we describe the features of this bacterium, together with the complete genome sequence, and annotation. The 2,160,700 bp long single replicon genome with its 2,053 protein-coding and 56 RNA genes is part of the Genomic Encyclopedia of Bacteria and Archaea project.

\section{Introduction}

Strain NGA ${ }^{\mathrm{T}}$ (= DSM $13181=$ ATCC BAA-54 = JCM 12221 ) is the type strain of Anaerobaculum mobile [1]. The genus, described for a thermophilic, citrate-fermenting anaerobe with $A$. thermoterrenum as the type species [2], was emended twice [1,3]. The third species is the recently described $A$. hydrogeniformans, forming $\mathrm{H}_{2}$ and acetate from glucose [3] while the other two species form $\mathrm{CO}_{2}$. A. mobile was isolated from a $10^{-7}$ dilution of an oleic acid-degrading consortium, originating from the sludge of a wool-scouring wastewater. Enrichment was performed in BCYT medium (basal BC medium [4] with tryptone and yeast extract), supplemented with crotonate [1]. Here we present a summary classification and a set of features for A. mobile $\mathrm{NGA}^{\mathrm{T}}$ together with the description of the complete genomic sequencing and annotation.

\section{Classification and features} 16S rRNA gene sequence analysis

A representative genomic $16 \mathrm{~S}$ rRNA gene sequence of $A$. mobile $\mathrm{NGA}^{\mathrm{T}}$ was compared using NCBI BLAST $[5,6]$ under default settings (e.g., considering only the high-scoring segment pairs (HSPs) from the best 250 hits) with the most recent release of the Greengenes database [7] and the relative frequencies of taxa and keywords (reduced to their stem [8]) were determined, weighted by BLAST scores. 
The most frequently occurring genera were Anaerobaculum (81.9\%), Acetomicrobium (9.9\%), Thermovirga (4.5\%) and Dethiosulfovibrio (3.6\%) (12 hits in total). Regarding the two hits to sequences from members of the species, the average identity within HSPs was $99.9 \%$, whereas the average coverage by HSPs was $100.0 \%$. Regarding the seven hits to sequences from other members of the genus, the average identity within HSPs was $97.1 \%$, whereas the average coverage by HSPs was $99.1 \%$. Among all other species, the one yielding the highest score was Acetomicrobium flavidum (FR733692), which corresponded to an identity of $99.8 \%$ and an HSP coverage of $100.0 \%$. (Note that the Greengenes database uses the INSDC (= EMBL/NCBI/DDBJ) annotation, which is not an authoritative source for nomenclature or classification.) The highest-scoring environmental sequence was FN436106 (Greengenes name 'Molekularbiologische Charakterisierung der bakteriellen Populationsdynamik eines thermophil betriebenen Biogasfermenters zur Vergaerung nachwachsender Rohstoffe thermophilic biogas reactor fed renewable biomass clone HAW-R60-B$\left.745 d-B C^{\prime}\right)$, which showed an identity of $99.9 \%$ and an HSP coverage of $99.9 \%$. The most frequently occurring keywords within the labels of all environmental samples which yielded hits were 'digest' (7.0\%), 'anaerob' (4.6\%), 'thermophil' (4.3\%), 'microbi' (3.4\%) and 'mesophil' (3.3\%) (238 hits in total). The most frequently occurring keywords within the labels of those environmental samples which yielded hits of a higher score than the highest scoring species were 'thermophil' (12.3\%), 'reactor' (8.8\%), 'bioga, fed' (7.0\%), 'bakteriellen, betriebenen, biogasferment, biomass, charakterisierung, molekularbiologisch, nachwachsend, populationsdynamik, renew, rohstoff, vergaerung' (5.3\%) and 'corn, microbi, silag, structur' (1.8\%) (4 hits in total).

A. flavidum is a species that was originally described without $16 \mathrm{~S}$ rRNA gene sequencing [9]. The sequence FR733692 was only recently been generated by DSMZ staff in the course of the Living-Tree Project [10], yielding an unexpected placement of the species within the Synergistetes. An assessment of whether a contamination or confusion of strains has occurred, or whether the currently available culture deposits are biologically identical to the original description but the classification of $A$. flavidum needs to be revised. This work is currently in progress. (R. Pukall (DSMZ), personal communication).
Figure 1 shows the phylogenetic neighborhood of A. mobile in a $16 \mathrm{~S}$ rRNA gene based tree. The sequences of the two identical 16S rRNA gene copies in the genome differ by one nucleotide from the previously published 16S rRNA gene sequence (AJ243189).

\section{Morphology and physiology}

The Gram-negative rod-shaped cells of $A$. mobile are straight (0.5-1.0 × 2.0-4.0 $\mu \mathrm{m}$; Figure 2), occurring singly or, predominantly in the exponential growth phase, in pairs. Longer cells (4.0-8.0 $\mu \mathrm{m})$ are observed in older cultures. Spores or sheath formation were never observed. Cells are moderately thermophilic; motile by means of a single flagellum inserted in the lateral region of the cell. On BCTC agar, colonies (1.0-1.5 $\mathrm{mm}$ in diameter) are white, lens shaped and with entire margins. Temperature and $\mathrm{pH}$ range for growth is between $35-65^{\circ} \mathrm{C}$ (optimum between 55 and $60^{\circ} \mathrm{C}$ ) and between 5.4 and 8.7 (optimum between 6.6 and 7.3), respectively. Growth occurs at $\mathrm{NaCl}$ concentrations of up to $1.5 \%$, with an optimum of $0.8 \%$.

Cells are strictly anaerobic, fermenting glucose, organic acids (pyruvate, tartrate and malate) and glycerol to acetate, $\mathrm{H}_{2}$ and, presumably, $\mathrm{CO}_{2}$ (the authors of [1] assumed that $2 \mathrm{~mol} \mathrm{CO}_{2}$ was produced per mol glucose, tartrate and malate degraded, and $1 \mathrm{~mol}$ $\mathrm{CO}_{2}$ was produced per mol pyruvate and glycerol degraded). Gluconate, L-malate, glycerol, tryptone, L-arginine, L-leucin, L-phenylalanine and starch are utilized [3]. No growth occurs on citrate, 2oxoglutarate, glutamate, mannose, pectin, lactose, xylose, galactose, maltose, sucrose, rhamnose, raffinose, malonate, lactate, succinate, xylan, dextrin, inulin, melibiose, adonitol, cellobiose, arabinose, polygalacturonate, cellulose, gelatin, butyrate or oleate [1], nor lactate, maltose, malonate, mannose, inositol and inulin [3].

In co-culture with Methanothermobacter thermautotrophicus glucose and malate degradation were enhanced. Crotonate is not fermented but used as an electron acceptor, reducing it to butyrate in the presence of yeast extract and tryptone, glucose, Casamino acids and leucine. Fumarate, acetate, sulfate, sulfite and nitrate are not used as electron acceptors in PY broth. The addition of crotonate, sulfur, cystine and thiosulfate enhances growth from tryptone and yeast extract, Casamino acids and glucose, as indicated by an increase in OD and by the production of reduction compounds (butyrate and sulfide, respectively) [1]. 


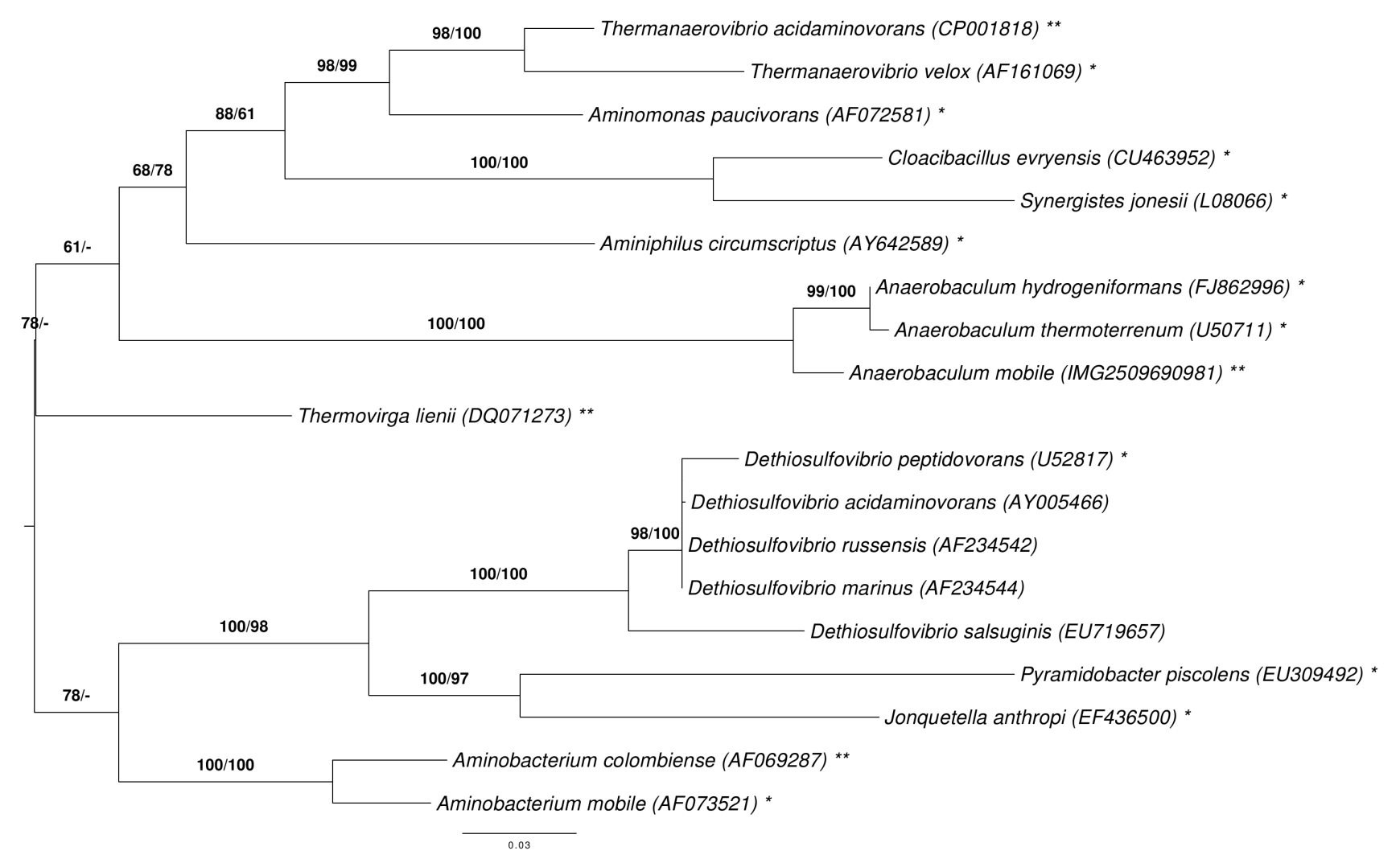

Figure 1. Phylogenetic tree highlighting the position of $A$. mobile relative to the type strains of the other species within the phylum 'Synergistetes'. The tree was inferred from 1,360 aligned characters [11,12] of the 16S rRNA gene sequence under the maximum likelihood (ML) criterion [13]. Rooting was done initially using the midpoint method [14] and then checked for its agreement with the current classification (Table 1). The branches are scaled in terms of the expected number of substitutions per site. Numbers adjacent to the branches are support values from 1,000 ML bootstrap replicates [15] (left) and from 1,000 maximum-parsimony bootstrap replicates [16] (right) if larger than 60\%. Lineages with type strain genome sequencing projects registered in GOLD [17] are labeled with one asterisk, those also listed as 'Complete and Published' with two asterisks [18-20]; the non-contiguous finished draft genomes of Aminomonas paucivorans [21] and Dethiosulfovibrio peptidovorans [22] lack the second asterisk.

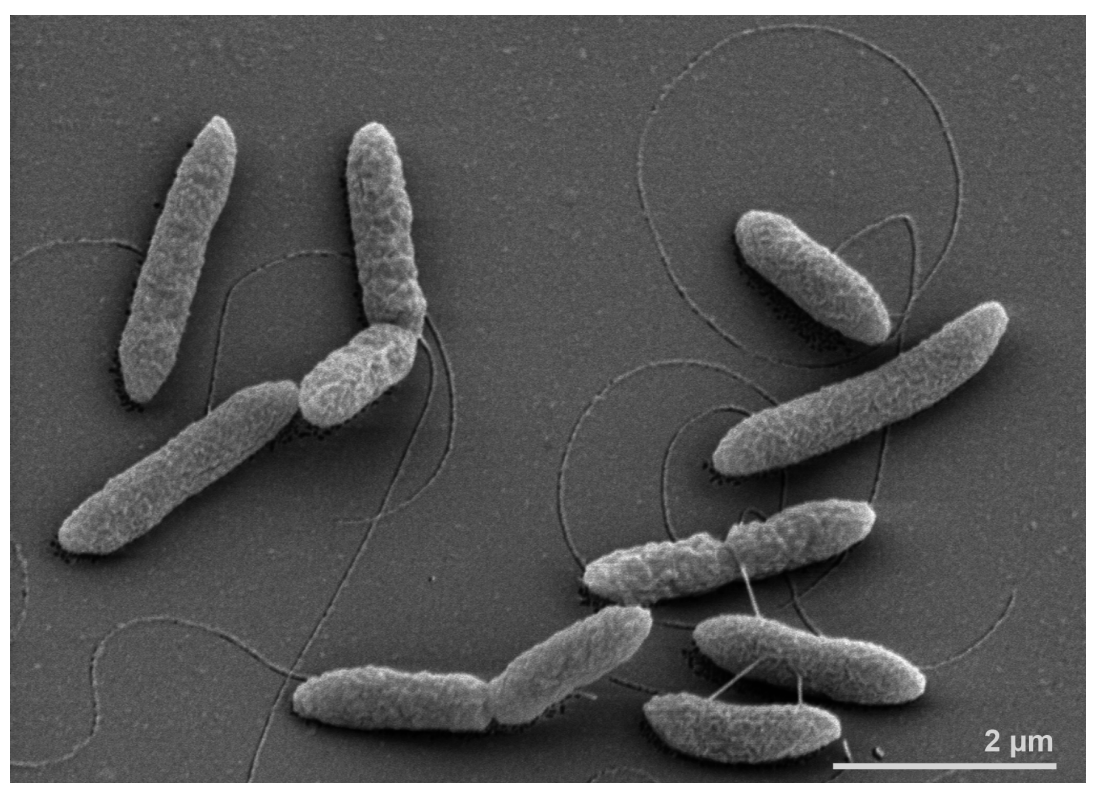

Figure 2. Scanning electron micrograph of $A$. mobile $\mathrm{NGA}^{\top}$ 


\section{Chemotaxonomy}

The cell wall is of the Gram-negative type as judged by transmission electron micrography [1]. Polar lipid composition includes diphosphatidylglycerol, phosphatidylglycerol, phosphatidylethanolamine, three unknown phos- pholipids and four unknown aminophospholipids [3]. Major fatty acids (>20\%) are iso- $\mathrm{C}_{15: 0}$ and iso$\mathrm{C}_{11: 0}$; iso- $\mathrm{C}_{13: 0} 3-\mathrm{OH}$ is found in smaller amounts $(<10 \%)$ [3]. The DNA G+C content was previously reported with $51.5 \mathrm{~mol} \%$ [1].

Table 1. Classification and general features of A. mobile $\mathrm{NGA}^{\top}$ according to the MIGS recommendations [23].

\begin{tabular}{|c|c|c|c|}
\hline MIGS ID & Property & Term & Evidence code \\
\hline & & Domain Bacteria & TAS [24] \\
\hline & & Phylum Synergistetes & TAS [25] \\
\hline & & Class Synergistia & TAS [25] \\
\hline & Current classification & Order Synergistales & TAS [25] \\
\hline & & Family Synergistaceae & TAS [25] \\
\hline & & Genus Anaerobaculum & TAS $[1,3,26]$ \\
\hline & & Species Anaerobaculum mobile & TAS [1] \\
\hline MIGS-7 & Subspecific genetic lineage (strain) & $\mathrm{NGA}^{\top}$ & TAS [1] \\
\hline \multirow[t]{8}{*}{ MIGS-12 } & Reference for biomaterial & Menes and Muxí 2002 & TAS [1] \\
\hline & Gram stain & Gram-negative & TAS [1] \\
\hline & Cell shape & rod-shaped & TAS [1] \\
\hline & Motility & motile & TAS [1] \\
\hline & Sporulation & non-sporulating & TAS [1] \\
\hline & Temperature range & $35-65^{\circ} \mathrm{C}$ & TAS [1] \\
\hline & Optimum temperature & $55-60^{\circ} \mathrm{C}$ & TAS [1] \\
\hline & Salinity & optimum growth at $0.8 \%$ & TAS [1] \\
\hline \multirow[t]{3}{*}{ MIGS-22 } & Relationship to oxygen & obligate anaerobe & TAS [1] \\
\hline & Carbon source & organic acids and carbohydrates & NAS \\
\hline & Energy metabolism & chemoorganotroph & TAS [1] \\
\hline MIGS-6 & Habitat & wastewater & TAS [1] \\
\hline MIGS-6.2 & $\mathrm{pH}$ & optimum $6.6-7.3$ & TAS 19] \\
\hline MIGS-15 & Biotic relationship & free living & TAS [1] \\
\hline MIGS-14 & Known pathogenicity & none & TAS [1] \\
\hline MIGS-16 & Specific host & not reported & \\
\hline MIGS-14 & Biosafety level & 1 & TAS [27] \\
\hline MIGS-19 & Trophic level & not reported & \\
\hline MIGS-23.1 & Isolation & wool-scouring wastewater treatment lagoon & TAS [1] \\
\hline MIGS-4 & Geographic location & Trinidad, Uruguay & TAS [1] \\
\hline MIGS-5 & Time of sample collection & November 1998 or earlier & NAS \\
\hline MIGS-4.1 & Latitude & -33.506 & TAS [1] \\
\hline MIGS-4.2 & Longitude & -56.889 & TAS [1] \\
\hline MIGS-4.3 & Depth & not reported & \\
\hline MIGS-4.4 & Altitude & not reported & \\
\hline
\end{tabular}

Evidence codes - TAS: Traceable Author Statement (i.e., a direct report exists in the literature); NAS: Non-traceable Author Statement (i.e., not directly observed for the living, isolated sample, but based on a generally accepted property for the species, or anecdotal evidence). Evidence codes are from of the Gene Ontology project [28]. 


\section{Genome sequencing and annotation \\ Genome project history}

This organism was selected for sequencing on the basis of its phylogenetic position [29], and is part of the Genomic Encyclopedia of Bacteria and Archaea project [30]. The genome project is deposited in the Genomes On Line Database [17] and the complete genome sequence is deposited in GenBank. Sequencing, finishing and annotation were performed by the DOE Joint Genome Institute (JGI) using state of the art sequencing technology [31]. A summary of the project information is shown in Table 2.

Table 2. Genome sequencing project information

\begin{tabular}{lll}
\hline MIGS ID & Property & Term \\
\hline MIGS-31 & Finishing quality & Finished \\
MIGS-28 & Libraries used & $\begin{array}{l}\text { Three genomic libraries: one 454 pyrosequence standard library, } \\
\text { one 454 PE libraries (11.0 kb insert size), one Illumina library }\end{array}$ \\
MIGS-29 & Sequencing platforms & Illumina GAii, 454 GS FLX Titanium \\
MIGS-31.2 & Sequencing coverage & $1,109.0 \times$ Illumina; $38.3 \times$ pyrosequence \\
MIGS-30 & Assemblers & Newbler version 2.3-PreRelease-6/30/2009, \\
& & Velvet version 1.0.13, phrap version SPS - 4.24 \\
MIGS-32 & Gene calling method & Prodigal 1.4, GenePRIMP \\
& INSDC ID & CP003198 \\
& GenBank Date of Release & June 14, 2012 \\
& GOLD ID & Gc02248 \\
& NCBI project ID & 53351 \\
& Database: IMG & 2509601011 \\
Source material identifier & DSM 13181 \\
& Project relevance & Tree of Life, GEBA \\
\hline
\end{tabular}

\section{Growth conditions and DNA isolation}

A mobile strain $\mathrm{NGA}^{\mathrm{T}}$, DSM 13181, was grown anaerobically in DSMZ medium 104b (PYX, medium) at $55^{\circ} \mathrm{C}$. DNA was isolated from 1-1.5 g of cell paste using a Jetflex Genomic DNA Purification Kit (GENOMED 600100) and following the standard protocol as recommended by the manufacturer with modification (an additional $10 \mu \mathrm{l}$ proteinase $\mathrm{K}$ digestion for cell lysis was added; $40 \mathrm{~min}$ incubation at $58^{\circ} \mathrm{C}$ ). DNA is available through the DNA Bank Network [32].

\section{Genome sequencing and assembly}

The genome was sequenced using a combination of Illumina and 454 sequencing platforms. All general aspects of library construction and sequencing can be found at the JGI website [33]. Pyrosequencing reads were assembled using the Newbler assembler (Roche). The initial Newbler assembly, consisting of 31 contigs in one scaffold, was converted into a phrap [34] assembly by making fake reads from the consensus, to collect the read pairs in the 454 paired end library. Illumina GAii sequencing data $(2,649.5 \mathrm{Mb})$ was assembled with Velvet [35] and the consensus sequences were shredded into $1.5 \mathrm{~kb}$ overlapped fake reads and assembled together with the 454 data. The $454 \mathrm{draft}$ assembly was based on $113.8 \mathrm{Mb} 454 \mathrm{draft}$ data and all of the 454 paired end data. Newbler parameters are -consed -a 50 -l 350 -g -m -ml 20. The Phred/Phrap/Consed software package [34] was used for sequence assembly and quality assessment in the subsequent finishing process. After the shotgun stage, reads were assembled with parallel phrap (High Performance Software, LLC). Possible mis-assemblies were corrected with gapResolution [33], Dupfinisher [36], or sequencing cloned bridging PCR fragments with subcloning. Gaps between contigs were closed by editing in Consed, by PCR and by Bubble PCR primer walks (J.-F. Chang, unpublished). A total of 68 additional reactions and 3 shatter libraries were 
necessary to close gaps and to raise the quality of the finished sequence. Illumina reads were also used to correct potential base errors and increase consensus quality using a software Polisher developed at JGI [37]. The error rate of the completed genome sequence is less than 1 in 100,000. Together, the combination of the Illumina and 454 sequencing platforms provided $1147.3 \times$ coverage of the genome. The final assembly contained 237,629 pyrosequence and 32,103,764 Illumina reads.

\section{Genome annotation}

Genes were identified using Prodigal [38] as part of the DOE-JGI genome annotation pipeline [39], followed by a round of manual curation using the JGI GenePRIMP pipeline [40]. The predicted CDSs were translated and used to search the National Center for Biotechnology Information (NCBI) nonredundant database, UniProt, TIGR-Fam, Pfam,
PRIAM, KEGG, COG, and InterPro databases. Additional gene prediction analysis and functional annotation was performed within the Integrated Microbial Genomes - Expert Review (IMG-ER) platform [41].

\section{Genome properties}

The genome statistics are provided in Table 3 and Figure 3 . The genome consists of one chromosome with a total length of $2,160,700$ bp and a G+C content of $48.0 \%$. Of the 2,109 genes predicted, 2,053 were protein-coding genes, and 56 RNAs; 34 pseudogenes were also identified. The majority of the protein-coding genes (85.7\%) were assigned a putative function while the remaining ones were annotated as hypothetical proteins. The distribution of genes into COGs functional categories is presented in Table 4.

Table 3. Genome Statistics

\begin{tabular}{lrr}
\hline Attribute & Number & \% of Total \\
\hline Genome size (bp) & $2,160,700$ & 100.00 \\
DNA coding region (bp) & $1,982,774$ & 91.77 \\
DNA G+C content (bp) & $1,036,446$ & 47.97 \\
Number of replicons & 1 & \\
Extrachromosomal elements & 0 & \\
Total genes & 2,109 & 100.00 \\
RNA genes & 56 & 2.66 \\
rRNA operons & 2 & \\
tRNA genes & 48 & 2.28 \\
Protein-coding genes & 2,053 & 97.34 \\
Pseudo genes & 34 & 1.61 \\
Genes with function prediction & 1,808 & 85.73 \\
Genes in paralog clusters & 881 & 41.77 \\
Genes assigned to COGs & 1,796 & 85.16 \\
Genes assigned Pfam domains & 1,809 & 85.78 \\
Genes with signal peptides & 276 & 13.09 \\
Genes with transmembrane helices & 487 & 23.09 \\
CRISPR repeats & 3 & \\
\hline
\end{tabular}




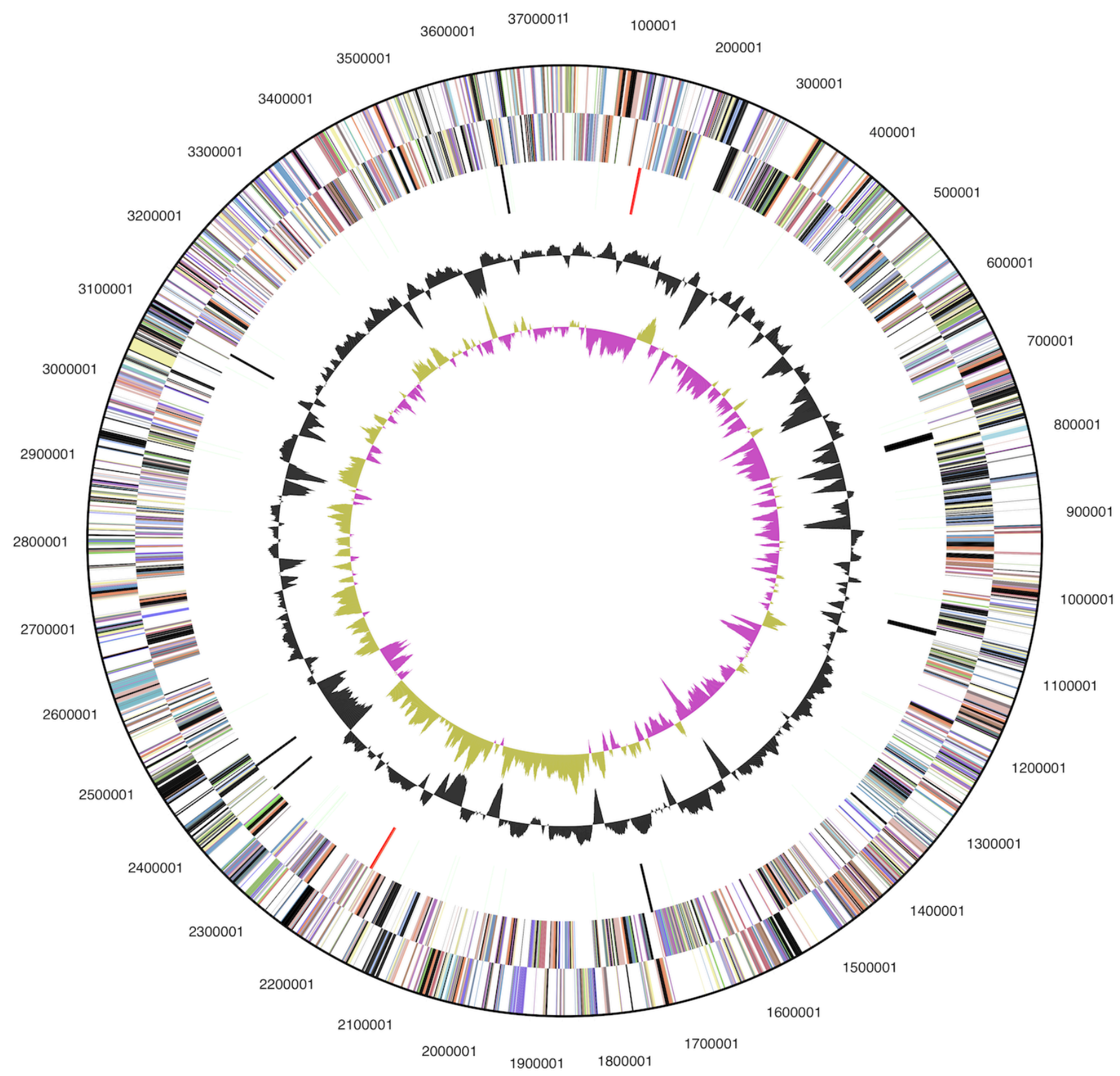

Figure 3. Graphical map of the chromosome. From outside to the center: Genes on forward strand (color by COG categories), Genes on reverse strand (color by COG categories), RNA genes (tRNAs green, rRNAs red, other RNAs black), GC content, GC skew (purple/olive). 
Table 4. Number of genes associated with the general COG functional categories

\begin{tabular}{crrll}
\hline Code & Value & \%age & Description \\
\hline J & 149 & 7.51 & Translation, ribosomal structure and biogenesis \\
A & 0 & 0.00 & RNA processing and modification \\
K & 86 & 4.34 & Transcription \\
L & 102 & 5.14 & Replication, recombination and repair \\
B & 0 & 0.00 & Chromatin structure and dynamics \\
D & 31 & 1.56 & Cell cycle control, cell division, chromosome partitioning \\
Y & 0 & 0.00 & Nuclear structure \\
V & 16 & 0.81 & Defense mechanisms \\
T & 56 & 2.82 & Signal transduction mechanisms \\
M & 117 & 5.90 & Cell wall/membrane/envelope biogenesis \\
N & 63 & 3.18 & Cell motility \\
Z & 0 & 0.00 & Cytoskeleton \\
W & 0 & 0.00 & Extracellular structures \\
U & 41 & 2.07 & Intracellular trafficking, secretion, and vesicular transport \\
O & 61 & 3.08 & Posttranslational modification, protein turnover, chaperones \\
C & 176 & 8.88 & Energy production and conversion \\
G & 131 & 6.61 & Carbohydrate transport and metabolism \\
E & 263 & 13.26 & Amino acid transport and metabolism \\
F & 69 & 3.48 & Nucleotide transport and metabolism \\
H & 85 & 4.29 & Coenzyme transport and metabolism \\
I & 34 & 1.71 & Lipid transport and metabolism \\
P & 92 & 4.64 & Inorganic ion transport and metabolism \\
Q & 36 & 1.82 & Secondary metabolites biosynthesis, transport and catabolism \\
R & 218 & 10.99 & General function prediction only \\
S & 157 & 7.92 & Function unknown \\
- & 313 & 14.84 & Not in COGs \\
\hline & & & \\
\hline
\end{tabular}

\section{Acknowledgements}

We would like to gratefully acknowledge the help of Maren Schröder for growing A. mobile cultures, Evelyne-Marie Brambilla for DNA extraction and quality control, and Cathrin Spröer and Rüdiger Pukall for helpful comments (all at DSMZ). This work was performed under the auspices of the US Department of Energy Office of Science, Biological and Environmental Research Program, and by the University of Cali- fornia, Lawrence Berkeley National Laboratory under contract No. DE-AC02-05CH11231, Lawrence Livermore National Laboratory under Contract No. DEAC52-07NA27344, and Los Alamos National Laboratory under contract No. DE-AC02-06NA25396, UTBattelle and Oak Ridge National Laboratory under contract DE-AC05-000R22725. 


\section{References}

1. Menes RJ, Muxi L. Anaerobaculum mobile sp. nov., a novel anaerobic, moderately thermophilic, peptide-fermenting bacterium that uses crotonate as an electron acceptor, and emended description of the genus Anaerobaculum. Int I Syst Evol Microbiol 2002; 52:157-164. PubMed

2. Rees GN, Pater BKC, Grassia GS, Sheehy AJ. Anaerobaculum thermoterrenum gen. nov., sp. nov., a novel, thermophilic bacterium which ferments citrate. Int J Syst Bacteriol 1997; 47:150154. PubMed http://dx.doi.org/10.1099/00207713-47-1-150

3. Maune MW, Tanner RS. Description of Anaerobaculum hydrogeniformans sp. nov., an anaerobe that produces hydrogen from glucose, and emended description of the genus Anaerobaculum. Int / Syst Evol Microbiol 2012; 62:832-838. PubMed http://dx.doi.org/10.1099/ijs.0.024349-0

4. Touzel JP, Albagnac G. Isolation and characterization of Methanococcus mazeii strain MC3. FEMS Microbiol Lett 1983; 16:241-245. http://dx.doi.org/10.1111/j.15746968.1983.tb00295.x

5. Altschul SF, Gish W, Miller W, Myers EW, Lipman DJ. Basic local alignment search tool. J Mol Biol 1990; 215:403-410. PubMed

6. Korf I, Yandell M, Bedell J. BLAST, O'Reilly, Sebastopol, 2003.

7. DeSantis TZ, Hugenholtz $\mathrm{P}$, Larsen $\mathrm{N}$, Rojas $\mathrm{M}$, Brodie EL, Keller K, Huber T, Dalevi D, Hu P, Andersen GL. Greengenes, a chimera-checked $16 \mathrm{~S}$ rRNA gene database and workbench compatible with ARB. Appl Environ Microbiol 2006; 72:5069-5072. PubMed http://dx.doi.org/10.1128/AEM.03006-05

8. Porter MF. An algorithm for suffix stripping. Program: electronic library and information systems 1980; 14:130-137.

9. Soutschek E, Winter J, Schindler F, Kandler O. Acetomicrobium flavidum, gen. nov., sp. nov., a thermophilic, anaerobic bacterium from sewage sludge, forming acetate, $\mathrm{CO}_{2}$ and $\mathrm{H}_{2}$ from glucose. Syst Appl Microbiol 1984; 5:377-390. http://dx.doi.org/10.1016/S0723-2020(84)80039$\underline{9}$

10. Munoz R, Yarza P, Ludwig W. Euzéby, Amann R, Schleifer K-H, Glöckner FO, Rosselló-Móra R. Release LTPs104 of the All-Species Living Tree.
Syst Appl Microbiol 2011; 34:169-170. PubMed http://dx.doi.org/10.1016/j.syapm.2011.03.001

11. Lee C, Grasso C, Sharlow MF. Multiple sequence alignment using partial order graphs.

Bioinformatics 2002; 18:452-464. PubMed http://dx.doi.org/10.1093/bioinformatics/18.3.452

12. Castresana J. Selection of conserved blocks from multiple alignments for their use in phylogenetic analysis. Mol Biol Evol 2000; 17:540-552.

PubMed

http://dx.doi.org/10.1093/oxfordjournals.molbev.a $\underline{026334}$

13. Stamatakis $A$, Hoover $\mathrm{P}$, Rougemont J. A rapid bootstrap algorithm for the RAxML web-servers. Syst Biol 2008; 57:758-771. PubMed http://dx.doi.org/10.1080/10635150802429642

14. Hess PN, De Moraes Russo CA. An empirical test of the midpoint rooting method. Biol / Linn Soc Lond 2007; 92:669-674.

http://dx.doi.org/10.1111/j.10958312.2007.00864.x

15. Pattengale ND, Alipour M, Bininda-Emonds ORP, Moret BME, Stamatakis A. How many bootstrap replicates are necessary? Lect Notes Comput Sci 2009; 5541:184-200. http://dx.doi.org/10.1007/978-3-642-02008-7_13

16. Swofford DL. PAUP*: Phylogenetic Analysis Using Parsimony (*and Other Methods), Version 4.0 b10. Sinauer Associates, Sunderland, 2002.

17. Pagani I, Liolios K, Jansson J, Chen IM, Smirnova T, Nosrat B, Markowitz VM, Kyrpides NC. The Genomes OnLine Database (GOLD) v.4: status of genomic and metagenomic projects and their associated metadata. Nucleic Acids Res 2012; 40:D571-D579. PubMed http://dx.doi.org/10.1093/nar/gkr1100

18. Chovatia M, Sikorski J, Schröder M, Lapidus A, Nolan M, Tice H, Del Rio TG, Copeland A, Cheng JF, Lucas S, et al. Complete genome sequence of Thermanaerovibrio acidaminovorans type strain $\left(S_{8} 883^{\top}\right)$. Stand Genomic Sci 2009; 1:254-261. PubMed http://dx.doi.org/10.4056/sigs.40645

19. Göker M, Saunders E, Lapidus A, Nolan M, Lucas S, Hammon N, Deshpande S, Cheng JF, Han C, Tapia R, et al. Genome sequence of the moderately thermophilic, amino-acid-degrading and sulfur-reducing bacterium Thermovirga lienii type strain (Cas60314 $)$. Stand Genomic Sci 2012; 6:230-239. PubMed 
http://dx.doi.org/10.4056/sigs.2726028

20. Chertkov O, Sikorski J, Brambilla E, Lapidus A, Copeland A, Del Rio TG, Nolan M, Lucas S, Chen $\mathrm{F}$, Tice $\mathrm{H}$, et al. Complete genome sequence of Aminobacterium colombiense type strain (ALA$1^{\top}$ ). Stand Genomic Sci 2010; 2:280-289. PubMed http://dx.doi.org/10.4056/sigs.902116

21. Pitluck S, Yasawong M, Held B, Lapidus A, Nolan M, Copeland A, Lucas S, Del Rio TG, Tice H, Cheng JF, et al. Non-contiguous finished genome sequence of Aminomonas paucivorans type strain $\left(\mathrm{GLU}-3^{\mathrm{T}}\right)$. Stand Genomic Sci 2009; 3:285-293. http://dx.doi.org/10.4056/sigs.1253298

22. LaButti K, Mayilraj S, Clum A, Lucas S, Del Rio TG, Nolan M, Tice H, Cheng JF, Pitluck S, Liolios $\mathrm{K}$, et al. Permanent draft genome sequence of Dethiosulfovibrio peptidovorans type strain (SEBR 4207 ${ }^{\top}$ ). Stand Genomic Sci 2010; 3:85-92. PubMed http://dx.doi.org/10.4056/sigs.1092865

23. Field D, Garrity G, Gray T, Morrison N, Selengut J, Sterk P, Tatusova T, Thomson N, Allen MJ, Angiuoli SV, et al. The minimum information about a genome sequence (MIGS) specification. Nat Biotechnol 2008; 26:541-547. PubMed http://dx.doi.org/10.1038/nbt1360

24. Woese CR, Kandler O, Wheelis ML. Towards a natural system of organisms: proposal for the domains Archaea, Bacteria, and Eucarya. Proc Natl Acad Sci USA 1990; 87:4576-4579. PubMed http://dx.doi.org/10.1073/pnas.87.12.4576

25. Jumas-Bilak E, Roudière L, Marchandin H. Description of 'Synergistetes' phyl. nov. and emended description of the phylum 'Deferribacteres' and of the family Syntrophomonadaceae, phylum 'Firmicutes'. Int J Syst Evol Microbiol 2009; 59:1028-1035. PubMed http://dx.doi.org/10.1099/ijs.0.006718-0

26. Rees GN, Patel BKC, Grassia GS, Sheehy AJ. Anaerobaculum thermoterrenum gen. nov., sp. nov., a novel, thermophilic bacterium which ferments citrate. Int / Syst Bacteriol 1997; 47:150154. PubMed http://dx.doi.org/10.1099/00207713-47-1-150

27. BAuA. 2010, Classification of bacteria and archaea in risk groups. http://www.baua.de TRBA 466, p. 24.

28. Ashburner M, Ball CA, Blake JA, Botstein D, Butler H, Cherry JM, Davis AP, Dolinski K, Dwight SS, Eppig JT, et al. Gene ontology: tool for the unification of biology. Nat Genet 2000; 25:25-29. PubMed http://dx.doi.org/10.1038/75556
29. Klenk HP, Göker M. En route to a genome-based classification of Archaea and Bacteria? Syst Appl Microbiol 2010; 33:175-182. PubMed http://dx.doi.org/10.1016/j.syapm.2010.03.003

30. Wu D, Hugenholtz P, Mavromatis K, Pukall R, Dalin E, Ivanova NN, Kunin V, Goodwin L, Wu $M$, Tindall BJ, et al. A phylogeny-driven genomic encyclopaedia of Bacteria and Archaea. Nature 2009; 462:1056-1060. PubMed http://dx.doi.org/10.1038/nature08656

31. Mavromatis K, Land ML, Brettin TS, Quest DJ, Copeland A, Clum A, Goodwin L, Woyke T, Lapidus A, Klenk HP, et al. The fast changing landscape of sequencing technologies and their impact on microbial genome assemblies and annotation. PLoS ONE 2012; 7:e48837; . PubMed http://dx.doi.org/10.1371/journal.pone.0048837

32. Gemeinholzer B, Dröge $G$, Zetzsche $H$, Haszprunar G, Klenk HP, Güntsch A, Berendsohn WG, Wägele JW. The DNA Bank Network: the start from a German initiative. Biopreserv Biobank 2011; 9:51-55. http://dx.doi.org/10.1089/bio.2010.0029

33. JGI website. http://www.jgi.doe.gov

34. The Phred/Phrap/Consed software package. http://www.phrap.com

35. Zerbino DR, Birney E. Velvet: algorithms for de novo short read assembly using de Bruijn graphs. Genome Res 2008; 18:821-829. PubMed http://dx.doi.org/10.1101/gr.074492.107

36. Han C, Chain P. Finishing repeat regions automatically with Dupfinisher. In: Proceeding of the 2006 international conference on bioinformatics \& computational biology. Arabnia HR, Valafar H (eds), CSREA Press. June 26-29, 2006: 141-146.

37. Lapidus A, LaButti K, Foster B, Lowry S, Trong S, Goltsman E. POLISHER: An effective tool for using ultra short reads in microbial genome assembly and finishing. AGBT, Marco Island, FL, 2008.

38. Hyatt D, Chen GL, LoCascio PF, Land ML, Larimer FW, Hauser LJ. Prodigal: prokaryotic gene recognition and translation initiation site identification. BMC Bioinformatics 2010; 11:119. PubMed http://dx.doi.org/10.1186/1471-2105-11$\underline{119}$

39. Mavromatis K, Ivanova NN, Chen IM, Szeto E, Markowitz VM, Kyrpides NC. The DOE-JGI Standard operating procedure for the annotations of microbial genomes. Stand Genomic Sci 2009; 
1:63-67. PubMed

http://dx.doi.org/10.4056/sigs.632

40. Pati A, Ivanova NN, Mikhailova N, Ovchinnikova G, Hooper SD, Lykidis A, Kyrpides NC.

GenePRIMP: a gene prediction improvement pipeline for prokaryotic genomes. Nat Methods 2010; 7:455-457. PubMed http://dx.doi.org/10.1038/nmeth.1457

41. Markowitz VM, Ivanova NN, Chen IMA, Chu K, Kyrpides NC. IMG ER: a system for microbial genome annotation expert review and curation. Bioinformatics 2009; 25:2271-2278. PubMed http://dx.doi.org/10.1093/bioinformatics/btp393 